\title{
The additive technology for obtaining thermal storages based on nanomodified materials
}

\author{
Alexander Shchegolkov ${ }^{1, *}$, Pavel Borovskikh ${ }^{2}$, and Larisa Shirshova ${ }^{3}$ \\ ${ }^{1}$ Tambov State Technical University, 392000, Tambov, Russia \\ ${ }^{2}$ Martin-Luther-Universität Halle-Wittenberg, Germany \\ ${ }^{3}$ Peoples Friendship University of Russia, 117198, Moscow, Russia
}

\begin{abstract}
The approaches for the implementation of an additive technology for obtaining heat accumulators are considered. The implementation of the provided technology can be realized on the standard 3D printers, which are aimed to obtain plastic materials. However, the software of the printers has to include adjusting analytical expressions, which take into account thermophysical properties of the heat-retaining materials. The analytical expressions have been derived by solving a mathematical model. The mathematical model contains the main data on thermophysical and mechanical and physical properties of the nanomodified material. These properties of the nanomodified material are defined during the experimental studies.
\end{abstract}

\section{Introduction}

An increase in efficiency of renewable energies is linked to the application of new innovative technologies and materials. Heat-retaining materials can be also used for an improvement of the element base of photovoltaics. It corresponds to the heat-retaining materials, which are capable for storing thermal energy of a phase transition [1-3]. In this case - for photovoltaics devices - the method of application is associated with an introduction of new functional capabilities (accumulation of thermal energy) and the stabilization of temperature condition. This type of the application allows obtaining both electric and thermal energy by use of the same device. In this case both economic and energy efficiencies increase. However, materials - capable to accumulate heat of a phase transition - lose their shape that is they melt and transit from a solid state into a liquid one. Thus, it hinders their application and leads to a need to use the auxiliary bodies and heat-exchanging elements. There is number of studies, in which potential improvements of such materials with a phase transition are researched, as for example an improvement of paraffin by introducing carbon nanotubes and grapheme into it [4-11]. The primary objective, which is set by authors of this paper, is related to the improvement of thermophysical properties, especially heat conduction. Other properties, which help to stabilize the shape, are not studied. Therein the relevant research goal is to study the issues of the nanomodification of paraffin and composite materials based on it in order to be able to apply them into solar power systems. Moreover, the aim is to obtain finished products, which have stable mechanical and physical properties in a wide temperature range and are suitable for using them in the additive (3D) technologies $[2,3]$. It is necessary to obtain a material with stable properties and to develop a technique for mathematical modeling in order to implement the additive technologies with a use of the nanomodified materials. The analytical functions will be derived from the mathematical model in order to use them in software for $3 \mathrm{D}$ printers.

\section{Additive technology}

Standard 3D printers, which are used for producing plastic products, can be utilized to implement the additive technology for obtaining heat storages based on nanomodified materials. There are number of techniques of the application of 3D technology for a creation of sophisticated products, which have channels for liquid [4,5]. The Fused Deposition Modeling (FDM) is employed to produce the thermal storage. A printing file of the STL format is created after the calculations - related to the modes of the thermal storage - have been performed. This allows segmenting the virtual model into layers. An expendable material in this printing technology is a composite polymer thread, which heats up to a melting temperature when it enters the extruder and is laid in doses through a nozzle on the working surface of $3 \mathrm{D}$ printer. Thus, a thermal storage - the form-lines and geometry of which precisely correspond to the virtual prototype - is being created layer by layer.

However, it is necessary to consider higher thermal capacity of heat-retaining materials and their mechanical and physical properties while creating heat-retaining modules. These properties are considered in specialized software. Analytical expressions - which take into account properties of heat-retaining material - are required for this purpose. These analytical expressions can be derived using approaches related to a solution of partial differential equations [14]. In the framework of the mathematical modeling it is necessary to know

\footnotetext{
* Corresponding author: Energynano@yandex.ru
} 
mechanical and physical properties of the nanomodified materials - planned to be applied - as well as distinctive features of these materials under conditions of various temperature regimes.

\subsection{Synthesis of samples of the nanomodified polymeric composites}

The samples - containing P-2 paraffin (Russia) and polyethylene (low pressure (PND) PE80B-275) (Russia) - were prepared for the research. The multi-layered cylindrical carbon nanotubes of the" Taunit M" type produced by JSC Nanotechcentre, Tambov, Russia -were employed as a modifying additive agent (Fig. 1) [12,13].

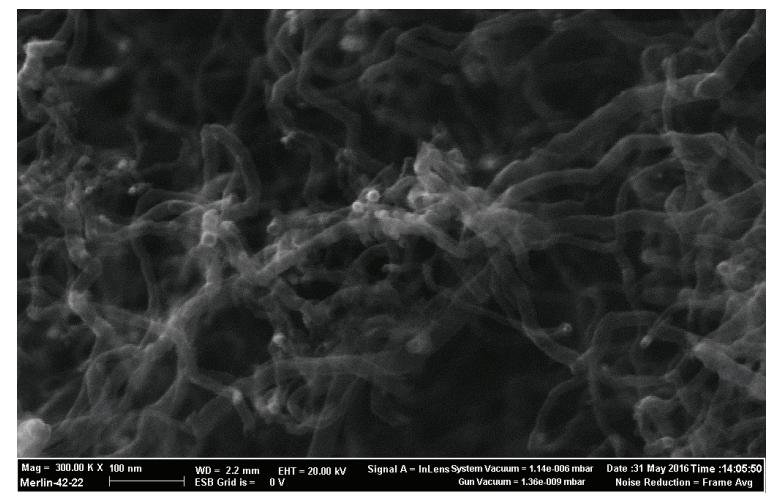

Fig. 1. SEM-image of the carbon nanotubes of the "Taunit" type

\subsection{The technique of a modification of a composite based on paraffin and polyethylene by carbon nanotubes}

The modification was carried out in 2 stages:

1) introducing carbon nanotubes - "M" into paraffin;

2) introducing a mixture of paraffin and carbon nanotubes into polyethylene by means of extrusion.

At the first stage the carbon nanotubes were placed into the silicone tank of the volume of $0,002 \mathrm{~m} 3$ and afterwards paraffin " $\mathrm{P} 2$ " heated to $80^{\circ} \mathrm{C}$ was added. This mixture was processed by ultrasound with a frequency of $22,5 \mathrm{kHz}$ within 1 hour while stirring it by means of a mechanical stirrer (200 rpm) and keeping temperature of $70^{\circ} \mathrm{C}$. As a result, black nontransparent material has been produced. Afterwards, the material was granulated by the press-granulator. The size of granules was in the range of 1 to $8 \mathrm{~mm}$.

The obtained granules were mixed (with mass ratio of 1:1) with polyethylene granules. Furthermore, these granules were placed into the hopper of the extruder. The extruder operates with a driving motor of $1,5 \mathrm{~kW}$ and a heater of $2 \mathrm{~kW}$. The produced material has been granulated after the extrusion.

\subsection{Methods for studying samples of the nanomodified polymeric composites}

The studies of thermophysical properties were conducted by means of "IT $-\lambda-400$ " device (measurement of heat conductivity, fig. 2 and 3) and "IT - S- 400" device (measurement of thermal capacity) within a monotonous warming mode with an average speed of $0,2^{\circ} \mathrm{C} / \mathrm{s}$ under adiabatic conditions.

The study of the density of granules was conducted by immersing them in distilled water and afterwards calculating the density with a consideration of displacement volume. The determination of bulk density was conducted by placing granules into the transparent vessel, shaking it within 2-3 minutes and then measuring the occupied volume.

The study of a temperature dependence of the volume was conducted in a measuring cylinder with volume of $0,001 \mathrm{~m} 3$. This measuring cylinder was put into a transparent cylinder of bigger volume $(0,01 \mathrm{~m} 3)$.

The free space between them was filled with the distilled water. The temperature of water was varied in increments of $2{ }^{\circ} \mathrm{C}$.

The study of durability was conducted by using a hydraulic press. The study of durability was conducted by using a hydraulic press. The granules were placed in a space between metal sheets.

\section{Results of the analysis of the nanomodified composite}

Figure 2 shows the results of a study on temperature dependence of thermal capacity of three materials: a composite (the nanomodified paraffin and polyethylene); paraffin modified by carbon nanotubes-"M"; and pure paraffin.

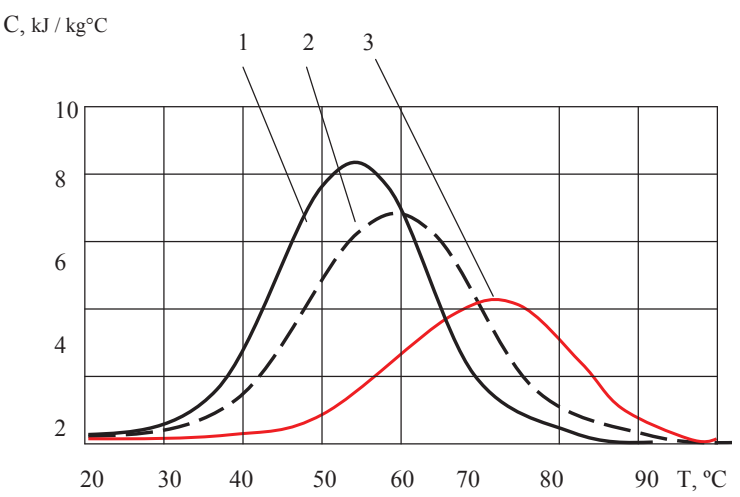

Fig. 2. Temperature dependence of thermal capacity of the nanomodified composite for 3D technology: 1 - paraffin; 2 nanomodified paraffin; 2 - paraffin + polyethylene (carbon nanotubes-“M").

Figure 3 shows the results of a study on temperature dependence of heat conductivity of composites modified by carbon nanotubes as well as pure paraffin. 


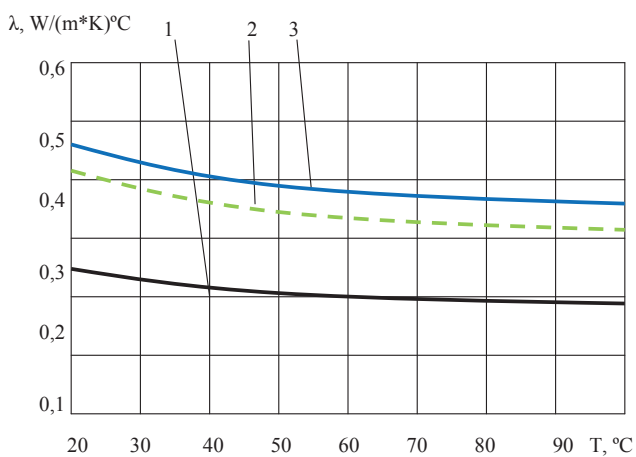

Fig. 3. Temperature dependence of heat conductivity of the nanomodified composite for 3D technology: 1 - paraffin; 2 paraffin + carbon nanotubes-"M", 2 - paraffin + polyethylene (carbon nanotubes-“M”)

In the figure 4 the temperature dependence of volume changes of composite materials is presented.

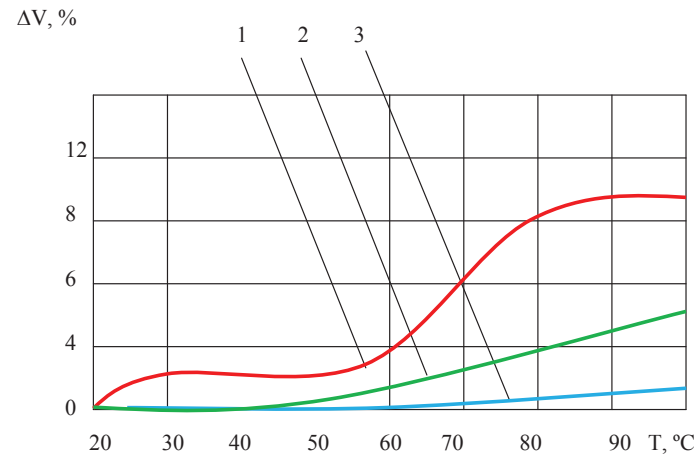

Fig. 4. Temperature dependence of volume changes of the nanomodified composite for 3D technology: 1 - paraffin; 2 nanomodified paraffin (carbon nanotubes-"M"); 3 - paraffin + polyethylene (carbon nanotubes-“M").

Mechanical and physical properties of the composite paraffin + polyethylene (carbon nanotubes-“M") for 3D technology are presented in the table 1.

Table 1. Mechanical and physical properties.

\begin{tabular}{|l|l|l|}
\hline № & $\begin{array}{l}\text { Mechanical and } \\
\text { physical properties }\end{array}$ & $\begin{array}{l}\text { paraffin + polyethylene } \\
\text { (carbon } \\
\text { "M”). }\end{array}$ \\
\hline 1 & bulk density, $\mathrm{kg} / \mathrm{m}^{3}$ & 7 \\
\hline 2 & real density, $\mathrm{kg} / \mathrm{m}^{3}$ & 1,2 \\
\hline 3 & $\begin{array}{l}\text { Durability at } \\
\text { compression of of } \\
\text { granules, MPa }\end{array}$ & 0,15 \\
\hline
\end{tabular}

\section{The technique for deriving analytical functions for 3D technology}

Within a framework of the additive technology it is necessary to consider the following situation: there is an overlaying of the material on a photo cell. From the perspective of physical model adaptation the photo cell is considered as an unbounded two-layer sheet with a functional source.

Problem of heat conductivity for an unbounded twolayer sheet with a functional source:

$$
\begin{gathered}
\frac{\partial t_{i}\left(x_{i}, \tau\right)}{\partial \tau}=a_{i} \frac{\partial^{2} t_{i}\left(x_{i}, \tau\right)}{\partial x_{i}^{2}}+\frac{q_{i}\left(x_{i}, \tau\right)}{c_{i} \rho_{i}} ; \quad i=1,2 ; \quad 0 \leq x_{i} \leq R_{i} \\
t_{i}\left(x_{i}, 0\right)=f_{i}\left(x_{i}\right) ; \\
\lambda_{1} \frac{\partial t_{1}(0, \tau)}{\partial x_{1}}-\alpha_{1}\left(t_{i}(0, \tau)-t_{c 1}\right)=0 ; \\
\lambda \frac{\partial t_{2}\left(R_{2}, \tau\right)}{\partial x_{2}}+\alpha_{2}\left(t_{2}\left(R_{2}, \tau\right)-t_{c 2}\right)=0 ; \\
\lambda_{1} \frac{\partial t_{1}\left(R_{1}, \tau\right)}{\partial x_{1}}=\lambda_{2} \frac{\partial t_{2}(0, \tau)}{\partial x_{2}} ; \\
t_{1}\left(R_{1}, \tau\right)=t_{2}(0, \tau) .
\end{gathered}
$$

Here, $\mathrm{x}$ - coordinate of thickness of a sheet, $\mathrm{m}$; $\tau$ - time, s;

$t_{i}\left(x_{i}, \tau\right)$ - temperature field of the $i$-th sheet layer, ${ }^{\circ} \mathrm{C}$; $R_{i}$ - thickness of the $i$-th sheet layer, m;

$a_{\mathrm{i}}-$ thermal diffusivity of a material of the $i$-th sheet layer, $\mathrm{m}^{2} / \mathrm{s}$;

$f_{i}\left(x_{i}\right)$ - initial temperature profile of the $i$-th sheet layer, ${ }^{\circ} \mathrm{C}$;

$\lambda_{i}, c_{i}$ - respectively heat conductivity, $\mathrm{W} /\left(\mathrm{m}^{\circ} \mathrm{C}\right)$, and thermal capacity, $\mathrm{J} /\left(\mathrm{kg}^{\circ} \mathrm{C}\right)$, of a material of the $i$-th sheet layer;

$\alpha_{i}$ - coefficient of convection heat transfer on a surface of the $i$-th sheet layer, $\mathrm{W} /\left(\mathrm{m}^{2 \circ} \mathrm{C}\right)$;

$t_{i}$ - environment temperature from the perspective of the $i$-th sheet layer, ${ }^{\circ} \mathrm{C}$.

The solution of the equations (1) - (6) is derived by the method of final integral transformations of the " $x$ " coordinate [14].

The solution is the following:

$$
\begin{gathered}
t_{i}\left(x_{i}, \tau\right)=P_{i}\left(x_{i}\right)+\sum_{n=1}^{\infty} \frac{T_{n}(\tau)}{N_{n}} S_{n}\left(x_{i}\right) ; \\
\bar{t}_{i}(\tau)=Q_{i}\left(x_{i}\right)+\sum_{n=1}^{\infty} \frac{T_{n}(\tau)}{N_{n}} Z_{i, n} ; \\
P_{1}\left(x_{1}\right)=\left(\frac{x_{1}}{\lambda_{1}}+\frac{1}{\alpha_{1}}\right) W+t_{c 1} ; \\
P_{2}\left(x_{2}\right)=\left(\frac{x_{2}}{\lambda_{2}}+\frac{R_{1}}{\lambda_{1}}+\frac{1}{\alpha_{1}}\right) W+t_{c 2} ; \\
Q_{1}\left(x_{1}\right)=\left(\frac{R_{1}}{2 \lambda_{1}}+\frac{1}{\alpha_{1}}\right) W+t_{c 1} ; \\
Q_{2}\left(x_{2}\right)=\left(\frac{R_{2}}{2 \lambda_{2}}+\frac{R_{1}}{\lambda_{1}}+\frac{1}{\alpha_{1}}\right) W+t_{c 2} ; \\
W=\frac{t_{c 2}-t_{c 1}}{\frac{R_{2}}{\alpha_{2}}+\frac{R_{1}}{\lambda_{2}}+\frac{1}{\alpha_{1}}} ;
\end{gathered}
$$




$$
\begin{gathered}
Z_{i, n}=\frac{\sqrt{a_{i}}}{\mu_{n} R_{i}}\left(\cos \left(\varphi_{i, n}\right)-\cos \left(\frac{\mu_{n} R}{\sqrt{a_{i}}}+\varphi_{i, n}\right)\right) ; \\
T_{n}(\tau)=\exp \left(-\mu_{n}^{2} \tau\right)\left(D_{n}+\int_{0}^{\tau} G\left(\mu_{n}, \tau\right) \exp \left(\mu_{n}^{2} \tau\right) d \tau\right) \\
D_{n}=\sum_{i=1}^{2} \frac{\lambda_{i}}{a_{i}} C_{i} \int_{0}^{R_{i}}\left(f_{i}\left(x_{i}\right)-P_{i}\left(x_{i}\right)\right) \sin \left(\frac{\mu_{n}}{\sqrt{a_{i}}} x_{i}+\varphi_{i, n}\right) d x_{i} ; \\
G\left(\mu_{n}, \tau\right)=\sum_{i=1}^{2} \frac{\lambda_{i}}{a_{i}} \int_{0}^{R_{i}} \frac{q_{i}\left(x_{i}, \tau\right)}{c_{i} \rho_{i}} \sin \left(\frac{\mu_{n}}{\sqrt{a_{i}}} x_{i}+\varphi_{i, n}\right) d x_{i} .
\end{gathered}
$$

$\mu_{n}$ - the $n$-th consecutive positive root of the equation

$$
\begin{gathered}
\frac{\lambda_{1} \sqrt{a_{2}}}{\lambda_{2} \sqrt{a_{1}}} \cos \left(\frac{\mu}{\sqrt{a_{1}}} R_{1}+\varphi_{1}\right) \sin \left(\varphi_{2}\right)+\sin \left(\frac{\mu}{\sqrt{a_{1}}} R_{1}+\varphi_{1}\right) \cos \left(\varphi_{2}\right)=0 ;(1 \\
\varphi_{1}=\operatorname{arctg}\left(\frac{\lambda_{1} \mu}{\alpha_{1} \sqrt{a_{1}}}\right) ; \\
\varphi_{2}=-R_{2} \frac{\mu}{\sqrt{a_{2}}}-\operatorname{arctg}\left(\frac{\lambda_{2} \mu}{\alpha_{2} \sqrt{a_{2}}}\right) ; \\
S_{n}\left(x_{i}\right)=C_{i, n} \sin \left(\frac{\mu_{n}}{\left.\sqrt{a_{i}} x_{i}+\varphi_{i, n}\right)} ;\right. \\
C_{1, n}=1 ; C_{2, n}=\frac{\sin \left(\frac{\mu_{n}}{\sqrt{a_{12}} R_{1}+\varphi_{1, n}}\right)}{\sin \left(\varphi_{2, n}\right)} ; \\
N_{n}=\sum_{i=1}^{2} \frac{\lambda_{i}}{a_{i}} C_{i}^{2}\left(\frac{\mu_{n}}{\sqrt{a_{i}}} R_{i}-\frac{1}{2}\left(\sin \left(2\left(\frac{\mu_{n}}{\sqrt{a_{i}}} R_{i}+\varphi_{i, n}\right)\right)-\sin \left(2 \varphi_{i, n}\right)\right)\right) ;
\end{gathered}
$$

\section{Discussion of research results of the nanomodified composite characteristics for the additive technology}

Thus, the temperature dependence of heat capacity of the obtained material is studied (the maximum value is 5 $\mathrm{kJ} / \mathrm{kg}^{\circ} \mathrm{C}$ ) as well as a comparison of it with heat capacity of pure paraffin $\left(8,2 \mathrm{~kJ} / \mathrm{kg}^{\circ} \mathrm{C}\right)$ is carried out. The phase transition is followed by temperature changes of the volume. It is found out that synthesized composite has a smaller tendency of changing volume with an increase in temperature $(2 \%)$, than pure $(12 \%)$ and nanomodified paraffin $(5 \%)$.

The study shows that it is possible to create materials - which are able to accumulate thermal energy - through the introduction of a component, which has high heat capacity, as for example nanomodified paraffin. Along with that, a use of nanomodified paraffin promotes a distribution of carbon nanotubes - "M" in a composite material such as polyethylene.
The mathematical modeling technique allows receiving analytical expressions which then can be applied in software of $3 \mathrm{D}$ printers.

\section{Conclusions}

1. The thermophysical and mechanical and physical properties of the obtained material are studied. It is found out that an extreme value of the temperature dependence of heat capacity changes its position on a graph.

2. The produced composite material can be employed for creating thermal storages due to its ability for phase transitioning with warmth of $5 \mathrm{~kJ} / \mathrm{kg}^{\circ} \mathrm{C}$ and strength properties of $0,15 \mathrm{MPa}$.

3. The mathematical modeling technique is designed. This technique allows using analytical dependences for software of equipment, which realizes the additive principle of the creation of 3-dimensional models.

This paper was financially supported by the Ministry of Education and Science of the Russian Federation on the Program to improve the competitiveness of RUDN University among the world's leading research and education centers in the 2016-2020.

\section{References}

1. Barnatt $\mathrm{CH}$. Nottingham, createspace independent publishing platform, 276 (2013)

2. Evans B. New york, apress, 306 (2012)

3. Lushin, E.N. Petersburg state polytechnical university journal. Physics and mathematics, 2, 4 (2013)

4. N. Bhattacharjee, for a. Urrios, s. Kang, a. Folch, lab chip, 16 (2016)

5. A.K. AU, W. Huynh, L.F. Horowitz, A. Folch, Chem. Int. Ed. Engl, 55 (2016)

5. Coleman J.N., Khan U., Blau W.J., Gun'ko Y.K. Carbon. 44, 9 (2006)

6. Zeng J.L., Cao Z., Yang D.W., Sun L.X., Zhang L., 101, 1 (2009)

7. Fan, L., Khodadadi, J.M. A review. Renewable and sustainable energy reviews, 15 (2011)

8. Warzoha, R. J., Zhang, D., Feng, G., Fleischer, A. S. CARBON, 61 (2013)

9. Warzoha, R. J., Fleischer, A. S. Applied Energy, 154, (2015)

10. Li, M., Guo, Q., Nutt, S. Solar energy, 146, (2017)

11. Liu, X., Rao, Z. Thermochimica acta, 647, (2017)

12. T.P. Dyachkova, Yu.A. Khan, N.V. Orlova, S.V. Kondrashov, Transactions TSTU. 22, 232 (2016)

13. A. A. Aladinskiy, A. V. Rukhov, E. N. Tugolukov, T. P. Dyachkova, Transactions TSTU. 20, 572 (2014)

14. Tugolukov E. N. the Monograph - Moscow. Mashinostroyeniye publishing house,. 100 (2004) 